\title{
Experiencias clave de aprendizaje de adultos chilenos en situación de vulnerabilidad: el reconocimiento como motivo de aprendizaje*
}

\author{
Key learning experiences of Chilean adults in situations of vulnerability: \\ Recognition as a reason for learning
}

\author{
Antonia Valdés ${ }^{a}$, Borja Castro-Serrano ${ }^{b}$ \\ ${ }^{a}$ Universidad de Barcelona \\ Correo electrónico: valdesantonia@gmail.com \\ ${ }^{\mathrm{b}}$ Escuela de Trabajo Social, Facultad de Humanidades y Ciencias Sociales, Universidad Andrés Bello \\ Correo electrónico: francisco.castro@unab.cl
} \begin{abstract}
de exclusión social. El objetivo consistió en identificar los significados que tienen 63 chilenos, estudiantes en un Instituto de capacitación popular, sobre las experiencias de aprendizaje que más los han marcado. Metodológicamente, a través del análisis de experiencias de aprendizaje obtenidas cualitativamente en cuestionarios escritos, se indagan elementos de su sentido de reconocimiento como aprendices. Los resultados indican que la mayoría de las experiencias clave de aprendizaje se relacionan con motivos de búsqueda de reconocimiento. Estas experiencias identificadas permiten al sujeto conectarse con diferentes motivos de aprendizaje, cuestión ilustrada a través de viñetas. A partir de estos resultados, se propone una discusión que integra algunos conceptos como los de reconocimiento, alteridad y subjetividad. Se busca, así, contribuir en los procesos de reflexividad necesarios en toda investigación cualitativa.
\end{abstract}

Este artículo presenta un estudio centrado en experiencias clave de aprendizaje de adultos que viven en condición

Palabras claves: identidad de aprendiz, motivos, reconocimiento, ética.

\begin{abstract}
This article presents a study focused on key learning experiences of adults living in conditions of social exclusion. The objective was to identify the meanings that 63 Chileans, students at a popular training institute, have about the learning experiences that have marked them the most. Methodologically, through the qualitative analysis of vignettes collected through written questionnaires, we investigated elements of their sense of recognition as learners. The results indicate that most of the key learning experiences relate to searching for recognition themes. These identified experiences allow the subject to connect with different learning motives. In our discussion of findings, we integrate concepts such as recognition, alterity, and subjectivity, aiming to contribute to the processes of reflexivity required in all qualitative research.
\end{abstract}

Key words: Learner identity, motifs, recognition, ethics.

\footnotetext{
Nos gustaría agradecer al equipo de investigación sobre Identidad de aprendiz que se enmarca en GRINTIE (Grupo de Investigación sobre Interacción e Influencia Educativa) de la Universidad de Barcelona. A todos sus miembros, en especial al líder del equipo, Dr. César Coll Salvador. Todos ellos han aportado al trabajo de discusión y elaboración de los instrumentos y análisis de los datos. También agradecemos el apoyo de CONICYT a través de su programa de BECAS CHILE que ha apoyado a la primera autora en la realización de sus estudios de Doctorado en el marco del mencionado grupo de investigación.
} 
Estudios Pedagógicos XLIII, Nº 3: 325-339, 2017

EXPERIENCIAS CLAVE DE APRENDIZAJE DE ADULTOS CHILENOS EN SITUACIÓN DE VULNERABILIDAD:

EL RECONOCIMIENTO COMO MOTIVO DE APRENDIZAJE

\section{INTRODUCCIÓN}

Uno de los caminos definidos para avanzar en la inclusión social y en el mejoramiento de la calidad de vida de las sociedades Occidentales consiste en mejorar las oportunidades de aprendizaje de todos los ciudadanos. Para ello es necesario no sólo mejorar las prácticas educativas, sino también fortalecer el sentido de reconocimiento de quienes participan en instancias de aprendizaje. Es decir, facilitar experiencias en las que los objetivos de aprendizaje se encuentren alineados con los motivos de aprendizaje en el sentido propuesto por Leontiev (2005). De ese modo, se integran dimensiones afectivas y prácticas inherentes a toda actividad de aprendizaje; pero, además, se reconoce que el otro reconfigura su subjetividad, entregándole autoría y autoridad en su proceso y narrativa de aprendizaje. Esto es particularmente importante cuando se trata de adultos que han "fracasado" en su trayectoria educativa formal y se encuentran en momentos de transición o de re-incorporación al sistema educativo. Más aún al ser personas cuya subjetividad (González-Rey, 2010) se encuentra permeada desde las experiencias de exclusión social y pobreza.

En esta línea es que se propone el concepto de «experiencia clave de aprendizaje» entendida como una experiencia subjetiva de aprendizaje especialmente significativa desde el punto de vista del sujeto. Algunos de los elementos que la caracterizan es que implica una toma de conciencia, una ruptura en la cadena de significados asociados al modo como se reconoce en cuanto aprendiz. Es un concepto que se enmarca en el enfoque socio constructivista, entendiendo el aprendizaje como un proceso en que se construyen significados y se atribuye sentido a través de la participación en actividades (Coll, 1988; Lave \& Wenger, 1991).

Este terreno conceptual permite reflexionar sobre el rol del otro y del reconocimiento en la propia subjetividad. Se introduce el concepto de "alteridad" que explora el tema de los actos de reconocimiento para repensar lo que ocurre a nivel intersubjetivo. Esta intersubjetividad hace un viraje desde la noción de Sujeto centrado en sí mismo hacia un Sujeto fisurado que en un paso posterior puede construir un sentido de reconocimiento a nivel intra-subjetivo. Se propone, finalmente, una reflexión filosófica que reescribe en clave ética la subjetividad y se pregunta por el tema del otro y lo Otro (Lévinas, 2006) en el proceso de aprender y de reconocerse como un aprendiz.

Este artículo se estructura en cuatro apartados: el primero desarrolla algunos conceptos teóricos que orientan la comprensión de las experiencias clave de aprendizaje como una herramienta analítica que articula la construcción de sentido del aprendizaje y el reconocimiento del sujeto como un aprendiz de determinadas características. El segundo describe los aspectos metodológicos del estudio llevado a cabo en Santiago de Chile. El tercer comenta los principales resultados de las construcciones discursivas que hacen 63 estudiantes del Instituto de Formación y Capacitación Popular (INFOCAP) acerca de sus experiencias clave de aprendizaje. Finalmente, se propone una discusión que reflexiona en torno a las implicancias educativas y éticas que emergen al abordar el tema de los motivos en los adultos que se reinsertan a espacios educativos formales. Se utilizan algunos conceptos como el de alteridad para examinar cómo el tema del reconocimiento se juega tanto en las instancias de enseñanza y aprendizaje como en las instancias de investigación. Se busca contribuir en los procesos de reflexividad necesarios en toda investigación cualitativa (Willig, 2013). 


\section{ANTECEDENTES TEÓRICOS}

El enfoque de «Aprendizaje durante toda la vida» propone que el aprendizaje no puede limitarse a una determinada edad, lugar ni tiempo. En este sentido es que coincide con la visión de una sociedad educativa en la que cada persona tiene la oportunidad de aprender y de desarrollar todas sus capacidades (Delors, 1996). Para alcanzar esta meta y para combatir la enorme desigualdad social es que se necesitan no sólo más y mejores oportunidades de aprendizaje. También es imperativo que fortalezcamos a los aprendices. En particular, que las personas se reconozcan como aprendices capaces y motivados por aprender en diferentes contextos a lo largo del tiempo (Biesta, Field, Hodkinson, Macleod \& Goodson, 2011).

Los esfuerzos actuales en materia de política pública educativa tanto en el plano nacional como internacional, buscan reconocer los innumerables espacios y nichos de aprendizaje que enfrentamos en una época marcada por la pérdida de estabilidad y difusión de fronteras entre lo público y lo privado, y entre lo formal e informal (Barnett, 2011). Nuevos desafíos aparecen también en torno a la generación de dispositivos que atiendan a la masa de sujetos que se reinserta en espacios de aprendizaje formal después de experiencias de fracaso o deserción escolar. En efecto, una de las preocupaciones constantes de la Unión Europea, por citar sólo un ejemplo, es el aprendizaje adulto y los modos en que se reengancha a quienes necesitan nuevas oportunidades educativas. Respecto a la situación chilena, podemos decir:

En el caso de Chile, así como en otros países de la región, las cifras dan cuentan de la concentración del ingreso, la creciente desigualdad y la desventaja económica de sectores de la sociedad producto del modelo de desarrollo impulsado: el neoliberalismo. Esta situación ha traído consecuencias en el ámbito de la salud física y mental, en la educación y en los problemas sociales propios de una gran población mundial, impulsando una crítica generalizada hacia el modelo imperante (Castro-Serrano, Flotts de los Hoyos, Valenzuela Parada y Vidal Molina, 2014: 134).

Este conjunto de elementos nos plantea la necesidad de abordar la complejidad del problema tomando en cuenta artefactos conceptuales que iluminen modos de conectar con la experiencia y subjetividad de los aprendices. En particular, con el modo cómo los aprendices construyen sentido para seguir aprendiendo en medio de circunstancias especialmente adversas derivadas de su contexto de vulnerabilidad socio-económica (Coll, 2003; González-Rey, 2010).

En este artículo se reflexiona en dos ámbitos epistemológicos y fenomenológicos íntimamente relacionados: por una parte, la problemática de la identidad de aprendiz, y por otra, la de la reconstrucción de experiencias de aprendizajes entendidas como posibilidades de reconocimiento. En torno al concepto de identidad, se recurre a propuestas derivadas del enfoque socio-constructivista. Específicamente se aborda el modelo de Identidad de Aprendiz desarrollado por Coll y Falsafi (2010). Para las experiencias de aprendizaje y su relación con el reconocimiento (Honneth, 1997; Castro-Serrano et al., 2014), se utilizan algunas ideas relacionadas con el concepto de motivos propuesto por Leontiev (1978; 2005) y algunos conceptos de Lévinas (2000; 2003; 2006) y de González-Rey (2010) que exploran la resignificación de la subjetividad. 


\subsection{LA IDENTIDAD DE APRENDIZ COMO CONSTRUCTO ANALÍTICO Y FENOMENOLÓGICO}

Inspirado en un marco sociocultural, se comprende el concepto de identidad enraizado en las prácticas culturales y en las actividades específicas en las que se despliega. Es decir, relevando su carácter dinámico y situado. En esta línea es que identidad y aprendizaje van indisolublemente unidos. A través de nuestra participación en actividades de aprendizaje vamos desarrollándonos y transformándonos. Es a través de nuestro aprender cotidiano junto a otros que vamos integrando determinadas comunidades. Al mismo tiempo, nuestra identidad nutre y media nuestros aprendizajes (Coll \& Falsafi, 2010; Lave \& Wenger, 1991; Wortham, 2004).

Durante la última década, hemos constatado un importante incremento en los estudios sobre identidad y su relación con el aprendizaje y esta tendencia tiene sólidas razones: el concepto de identidad es un poderoso lente o herramienta analítica (Sfard \& Prusak, 2005; Gee, 2000; Wortham, 2004). Ejemplos del uso de este concepto incluyen estudios sobre la influencia de identidades particulares (etnia, género) en el proceso de aprender contenidos específicos, por ejemplo, matemáticas y adquisición de un segundo idioma (Black, Williams, Hernandez-Martinez, Davis \& Wake, 2010).

Estos estudios son valiosas contribuciones que apuntan en la dirección de comprender la relación entre la identidad y el aprendizaje. Sin embargo, el estudio del concepto de la identidad de aprendiz (Coll \& Falsafi, 2010) requiere de mayores esfuerzos, pues aborda precisamente la identidad funcional en todos los contextos de aprendizaje. Es decir, aquella que se pone en juego cada vez que enfrentamos una nueva situación de aprendizaje.

En este sentido es que el Modelo de Identidad de Aprendiz (IdA) propuesto por Coll y sus colaboradores, es relevante como herramienta analítica y fenomenológica. En su modelo, proponen la identidad de aprendiz como: “(...) the conceptual artifact that contains, connects and enables reflection over the emotional and cognitive processes of the experience of becoming and being a learner, in the past as well as in the present and the future" (Coll \& Falsafi, 2010: 219). De lo anterior se desprende que la identidad de aprendiz constituye la base de todos los otros tipos de identidad. Lo anterior se reafirma al postular que la construcción del sentido de reconocimiento que tiene el sujeto sobre sí mismo como alguien más o menos capaz de aprender, se desprenden de tres elementos conceptuales claves: (1) la naturaleza discursiva de la identidad; (2) la construcción de la identidad a través de las actividades y prácticas sociales, y (3) el reconocimiento como un ingrediente esencial del proceso de construcción de identidad.

Los elementos y procesos involucrados en la identidad de aprendiz surgen a partir de las características y condiciones de la actividad entendidas desde el marco de la Teoría de la Actividad (Leontiev, 1978). Los procesos intra-psicológicos que interactúan con las condiciones de la actividad son las emociones que se entienden también como un componente de la actividad. La construcción de la identidad tiene lugar, entonces, en la actividad y está medida por el discurso, pero exige de reconocimiento pues en la medida en que no hay otros que nos reconozcan, no hay reconocimiento de uno mismo (ver figura 1). 
Figura I. Diagrama de elementos que intervienen en la construcción de la Identidad de Aprendiz (IdA).

\section{Elementos del Modelo de Identidad de Aprendiz}

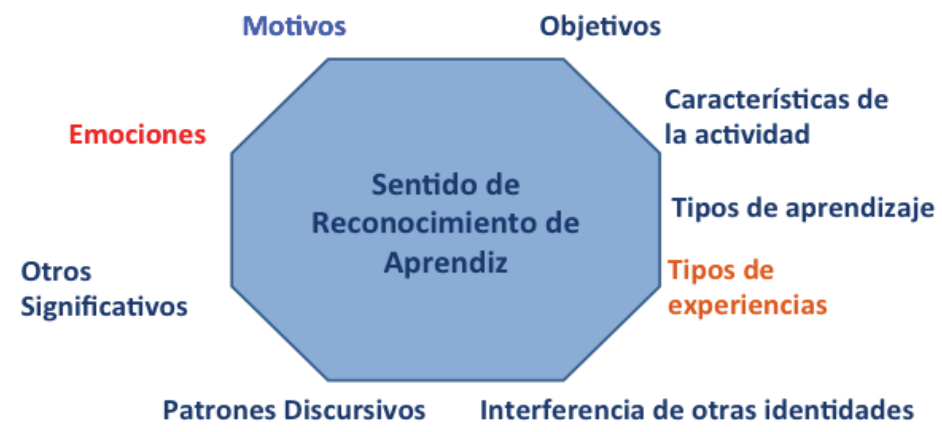

(Fuente: Falsafi, 2010: 265)

Dicho de otro modo, es a través de la experiencia de ser reconocido por otros (dimensión experiencial) que vamos dando sentido a la participación en las actividades de aprendizaje, lo que refleja la función interpsicológica que cumple esta identidad. Al estar insertos en la actividad, se está también en función de los objetivos y motivos que la impulsan y la orientan. En definitiva, los elementos que afectan el sentido de reconocimiento de los aprendices son los motivos y objetivos que cruzan la experiencia, las características de la actividad, los diferentes tipos de actividad que posibilitan la construcción de la identidad de aprendiz, los patrones discursivos y su rol en los significados de los sujetos, el rol de los otros significativos en el proceso de reconocimiento y de reconstrucción de significados y las emociones implicadas en este proceso.

\subsection{LAS EXPERIENCIAS CLAVE DE APRENDIZAJE COMO MÉTODO DE INDAGACIÓN Y DE CO- CONSTRUCCIÓN DE RECONOCIMIENTO}

En base a lo anterior, las experiencias clave de aprendizaje son reconstrucciones discursivas sobre experiencias que inciden fuertemente en el modo como los aprendices se reconocen. Inciden en el sentido de reconocimiento y también en los motivos de aprendizaje. Pueden cuestionar o confirmar los motivos que tiene el sujeto para aprender. Esto tiene implicancias prácticas en el sentido que genera disposiciones y posicionamientos específicos frente a actividades cuya meta es aprender o que tienen como resultado el aprendizaje.

Las experiencias subjetivas son la materia prima para la construcción de toda identidad. Es a través de la reconstrucción de los significados de experiencias que se construyen los significados también de nosotros mismos como personas, y en el caso que nos ocupa, como aprendices. Las experiencias clave de aprendizaje, constituyen, por lo tanto, una entrada privilegiada para conectar distintos significados y comprender cómo el sujeto se reconoce y se posiciona: cómo construye sentido en su propio proceso de aprendizaje. Son similares a conceptos planteados por la tradición biográfica, por ejemplo, los "episodios nucleares" y turning points propuestos por Denzin (1989) o McAdams, Josselson y Lieblich (2006). 
Sin embargo, el foco de interés principal no reside en el estudio de la coherencia de la identidad narrativa. Lo que interesa de estas experiencias es justamente ver cómo inciden en el sentido de reconocimiento y en el posicionamiento de los aprendices.

Algunos autores han investigado el impacto de las experiencias transformadoras en los contextos formales de educación (Woods, 1997; Yair, 2008). En estos estudios se aprecia cómo las experiencias que transforman a los sujetos pueden alterar incluso su vocación y futuras trayectorias de aprendizaje. Pero no se elabora mayormente en torno al proceso de transformación de significados, reconocimiento y posicionamiento que experimentan los aprendices. Nuestra investigación se orienta, justamente, a llenar este vacío y abordar tanto los procesos y funciones inter como intra psicológicos implicados en estas experiencias clave de aprendizaje.

\section{METODOLOGÍA}

El estudio es de carácter cualitativo interpretativo (Willig, 2013). Los resultados comentados en este artículo son parte de una investigación más amplia enmarcada en una línea de investigación sobre la construcción de la identidad de aprendiz. Entre los años 2011 a 2013 se recogieron datos en tres países diferentes (España, Brasil, Chile) explorando las experiencias de aprendizaje de adolescentes y adultos y su influencia sobre el modo cómo se reconocen en cuanto aprendices. La exploración se realizó a través de cuestionarios escritos y entrevistas en profundidad. En total, han participado más de 300 personas. En el caso del estudio citado en este artículo, corresponde a la fase de cuestionarios escritos de la muestra chilena. Para el análisis de los datos se realizó análisis de contenido, construyendo clusters temáticos y contrastando los datos con los elementos teóricos del modelo de IdA presentado anteriormente. Para el levantamiento de categorías y códigos, se llevó a cabo un proceso de construcción conjunta entre un equipo compuesto por seis investigadores. Luego se transcribieron todos los cuestionarios y se procedió a codificar utilizando el software Atlas.ti v.6. La codificación se realizó por duplas y en caso de discrepancias se acudió a un tercer juez. En este proceso recursivo de ir de los datos a la teoría y vice-versa, se fueron construyendo y ajustando códigos de modo de captar los elementos que articulan las experiencias significativas con el proceso de reconocerse como un determinado aprendiz.

La pregunta de investigación explorada en este artículo es: ¿Qué caracteriza los procesos de reconstrucción de motivos en las experiencias clave de aprendizaje de estos sujetos?

Se trabaja con estudiantes adultos que asisten al Instituto de Formación y Capacitación Popular (INFOCAP) ubicado en Santiago de Chile. Este Instituto recibe a personas que viven en condiciones de alta vulnerabilidad social. Los participantes del estudio fueron 63 estudiantes de este Centro cuya edad promedio era de 36 años. De ellos, 47 son mujeres y 16 hombres. Poco más de la mitad de los sujetos $(63 \%)$ posee educación secundaria terminada. El 5\% no ha completado la educación primaria y un $11 \%$ sólo ha completado la primaria. El promedio de años de escolaridad de los participantes son 10, y el de padres y madres de los participantes es de 8 años. Los estudiantes fueron invitados a participar voluntariamente en el estudio, informándoles los objetivos del mismo. Se aseguró la confidencialidad en cuanto a la reserva de sus datos personales. Esto incluye el cambio de sus nombres en el manejo de los datos transcritos y el consentimiento firmado sobre su participación. Para la 
administración del cuestionario se contó con un equipo mixto de investigadores tanto de la propia institución como externos que respondieron las dudas y estuvieron atentos para consultas y comentarios. Por último, se ofreció un espacio de debriefing a los participantes que así lo requirieran. Se contó con una psicóloga perteneciente al equipo de investigadores que estuvo disponible para entrevistas después de administrados los cuestionarios y se les recordó que frente a cualquier inquietud o comentario podían acudir también al servicio de asistencia psicológica que atiende en la misma institución.

En lo que respecta al instrumento, se utilizó un cuestionario semi-abierto elaborado por el equipo participante. El cuestionario constaba de tres partes. La primera indagaba datos socio demográficos, considerando datos como: escolaridad del participante y de sus padres, y ocupación actual. En la segunda se les pedía a los sujetos que identificaran y describieran tres experiencias clave de aprendizaje: las que fuesen especialmente significativas y que los hubiesen marcado en el modo cómo enfrentan el aprendizaje. Además de la descripción de la experiencia, debían responder preguntas que indagaban por las características teóricamente relevantes, tales como: el contexto en que había tenido lugar la experiencia; el interés y motivos para participar; la influencia que había tenido la experiencia en su vida. La tercera parte exploraba el modo cómo se reconocían y definían en cuanto aprendices tanto en las descripciones sobre sus fortalezas como en la identificación de los elementos de estructura de participación y de tipos de tareas que les facilitaban y obstaculizaban el aprendizaje.

El proceso de análisis de los datos tuvo varios pasos. Primero se procedió a la lectura y la construcción de códigos, la cual estuvo enmarcada en los elementos teóricos relevantes para el análisis. En este caso en particular, aquellos elementos referidos a los motivos de aprendizaje y al reconocimiento. Segundo, el proceso fue realizado de manera colaborativa entre varios investigadores siendo un proceso circular y recursivo. Por último, nuevas categorías y códigos surgieron de las discusiones teóricas a medida en que se procesaban los datos.

\section{RESULTADOS}

Exploraremos los resultados en torno a la siguiente pregunta: ¿Qué caracteriza los procesos de reconstrucción de motivos en las experiencias clave de aprendizaje de estos sujetos?

Es importante aclarar que comprendemos los motivos desde la conceptualización de la Teoría de la Actividad propuesta por Leontiev $(1978 ; 2005)$ y derivada de los aportes de Vygotsky. En esta propuesta, se conceptualiza la actividad dirigida por sus objetos y por los motivos de los individuos. Los motivos impulsan la participación de los sujetos en actividades específicas, pero co-existen en múltiples niveles simultáneos. Además, puede darse que los motivos del sujeto no coincidan con los objetivos de una actividad específica. Del mismo modo, puede ocurrir que en una misma actividad coexistan motivos personales de diferente nivel, que responden a metas más cercanas o lejanas. En este sentido y congruente con los postulados vygotskianos, se comprende que todo análisis de cualquier actividad contiene tanto la dimensión individual como la social: que no hay intra-subjetividad sin intersubjetividad. Algunos motivos serán, por lo tanto, más simples, específicos e inmediatos, y otros tendrán que ver con motivos de orden superior proyectados en el tiempo y que permiten al sujeto pensarse en el futuro. Esta es también la comprensión de motivos propuesta en el modelo Identidad de Aprendiz ya mencionado. Por lo tanto, la 
idea es explorar los motivos para captar mejor qué va ocurriendo con el modo como los sujetos se van reconociendo en cuanto aprendices.

Al examinar los tipos de motivos descritos por los participantes de este estudio aparece con claridad que la reconstrucción de motivos está íntimamente ligada con los procesos de reconocimiento. Esta es una relación recíproca y compleja, porque efectivamente el ser reconocido como un aprendiz de determinadas características puede ser un motivo en sí mismo (Falsafi, 2010). Al analizar los motivos, vemos que en la mayoría de las experiencias (71\% del total) el motivo se refiere al reconocimiento. Es decir, lo que motivaba al sujeto a participar en esa experiencia tenía que ver con que un otro lo reconociera de determinada manera y/o con que él mismo se reconociera con determinadas características y en un posicionamiento específico. Veamos la siguiente viñeta como ejemplo:

Ejemplo n¹. Experiencia Clave de aprendizaje: "Cuando salí de 4ºmedio" (Ana). Título: "Cuando salí de $4^{\circ}$ medio".

Cuando salí de $4^{\circ}$ medio, ya que me costó mucho porque repetí un año, pero logré salir del colegio, aunque tuve que hacer dos cursos en uno, porque había nacido mi hijo y aparte de estudiar tenía que ver a mi hijo. Cuéntanos... ¿Qué edad tenías? 21 ¿Dónde ocurrió? En el colegio ¿Había otras personas importantes en esa experiencia? Mi padre y madre. ¿Hubo alguien especial? Madre. ¿Por qué fue especial esa persona? Mi madre fue muy importante porque yo mientras que iba a estudiar ella me cuidaba a mi hijo. ¿Qué te llama la atención de esa experiencia? Que fui capaz de lograrlo, aunque de primera no me apoyaron mucho. ¿Hubo algo que te motivara especialmente? Si, salir adelante para trabajar y darle un buen futuro a mi hijo. ¿Cómo te sentiste? Me sentí feliz ya que deseaba sacar mi $4^{\circ}$ medio. ¿Cómo te sientes ahora? Me siento orgullosa de haberlo logrado. Me sentí contenta de que mi mamá se sintiera orgullosa de mi. ¿Te ha influido esa experiencia? ¿Cómo? Ha influido, porque ya el hecho de tener $4^{\circ}$ medio como que hasta el trato cambió.

Como puede apreciarse en el ejemplo de Ana, la experiencia escogida contiene motivos de diferente nivel. Un motivo, enunciado en el título, tiene que ver con lo acreditativo: "salir de 4to medio", que en Chile significa terminar la educación secundaria. Ese paso ofrece, a su vez, una posibilidad de cambio de status pues las posibilidades laborales son muy diferentes entre quienes han terminado la enseñanza secundaria. Esto se ve reflejado en el comentario final en que Ana identifica que incluso ha cambiado el trato que le dan por "tener 4to medio". Además, podemos apreciar un motivo de orden superior que tiene que ver con darle un buen futuro a su hijo y "salir adelante". El sentido de reconocimiento de Ana se construye a partir de actos de reconocimiento concretos como puede ser la obtención del diploma de egreso de la educación secundaria. Pero también, intervienen actos de reconocimiento vinculados íntimamente a sus otros significativos. Ella se siente feliz también de haber logrado que su madre estuviese orgullosa de ella. En este sentido es que los otros significativos prestan no sólo ayudas concretas para posibilitar el aprendizaje (la madre le cuidaba a su hijo para que pudiese estudiar), también entregan actos de reconocimiento que permiten al sujeto reconocerse como un aprendiz competente. 
Ejemplo $n^{\circ}$ 2. Experiencia Clave de Aprendizaje: "En la formación de mi oficio" (Pedro). Título: "En la formación de mi oficio".

INFOCAP creo que me enseñó mi oficio con mucho respeto, creyeron en mí y me dieron la mejor formación. Cuéntanos... ¿Qué edad tenías? 36 ¿Dónde ocurrió? En INFOCAP ¿Había otras personas importantes en esa experiencia? Amigos, Maestros, Compañeros. ¿Hubo alguien especial? Mis maestros. ¿Por qué fue especial esa persona? Mis maestros, creo que ellos fueron fundamentales para mí, gracias al apoyo de ellos pude terminar mi proceso. ¿Qué te llama la atención de esa experiencia? Si el respeto hacia nosotros por ser adultos que volvían a estudiar y el respeto hacia nuestra historia de vida. ¿Hubo algo que te motivara especialmente? Si, creer en mí, en mis capacidades, mis maestros influyeron en ese proceso. ¿Cómo te sentiste? Como una persona valorizada, capaz de lograr todas mis expectativas. ¿Cómo te sientes ahora? Me siento muy grato y siento que el futuro solo depende de mí, las herramientas que poseo. Me sentí, muy capaz, muy potenciado a seguir por mis maestros. ¿Te ha influido esa experiencia? ¿Cómo? Si, por ejemplo, puedo entender mejor los temas que se plantean.

El caso de la experiencia anterior, comentada por Pedro, nos sirve para ilustrar uno de los resultados encontrados en nuestra exploración de estas experiencias clave de aprendizaje. Se trata de las características que emergen cuando quien brinda el reconocimiento es el maestro. La mayoría de las experiencias en que esto ocurre, nos encontramos con que el sujeto se reconoce movilizado por el respeto que tiene su profesor por él en cuanto subjetividad. Aquí hay una experiencia de sentirse reconocido desde lo que se es, desde la propia historia. Como dice Pedro: "respeto hacia nuestras historias de vida". En cada una de las experiencias en que aparecen estos maestros vemos que el sentido de reconocimiento del sujeto en cuanto alguien capaz de aprender se fortalece de manera importante. Emergen nuevos escenarios construidos ya desde ese sentirse visto y capaz como aprendiz. “(...) me siento como una persona valorizada”, “(...) el futuro sólo depende de mí, de las herramientas que poseo”. Se construye una relación de respeto desde la cual el reconocimiento se transforma en potente movilizador para el sujeto.

Ejemplo no3. Experiencia Clave de aprendizaje: "Aprender a no callarme cuando tengo que decir algo", Título: "Aprender a no callarme cuando tengo que decir algo" (José).

En que un día un jefe me tiró su gineta encima y hoy la personalidad que tomé en el INFOCAP y lo que he aprendido ya no me hace callarme al debatir con alguien. Cuéntanos... ¿Qué edad tenías? 27 ¿Dónde ocurrió? En el trabajo ¿Había otras personas importantes en esa experiencia? Compañeros. ¿Hubo alguien especial? Un compañero ¿Por qué fue especial esa persona? Porque escucha todo lo que digo. ¿Qué te llama la atención de esa experiencia? Si, que cuando uno habla con base a alguien y es de igual a igual debatiendo ya no te miran en menos. ¿Hubo algo que te motivara especialmente? Si, que el hombre me dijo que él no era como yo, que no necesitaba andar en micro o metro porque él tenía plata ¿Cómo te sentiste? En ese momento mal. ¿Cómo te sientes ahora? Ahora bien. ¿Te ha influido esa experiencia? ¿Cómo? Bien, porque más ganas le echo al trabajo y ya no me dejan callado cuando uno debate cosas.

Si examinamos la experiencia anterior de José, vemos que nuevamente la experiencia cargada emocionalmente ha significado un quiebre en los significados. Un jefe lo menosprecia y hace una diferencia entre ellos mediada porque uno de los dos tiene dinero y el otro no. Surge el aprendizaje como una herramienta que le da al sujeto la capacidad 
de enfrentar ese espacio de debate ya no desde el silencio, sino desde el que tiene algo valioso que decir. "Ya no me dejan callado". Puede plantearse que es a partir de ese acto de reconocimiento negativo realizado por el jefe, que se vuelve visible para el sujeto el reconocimiento como un motivo que lo impulsa a formarse.

Los resultados muestran que la gran mayoría de las experiencias compartidas por los sujetos están relacionadas con emociones intensas y positivas. Esto ocurre tanto respecto de las emociones que asocian a la experiencia como a las emociones asociadas específicamente a los actos de reconocimiento que identifican. La tendencia global es relacionar actos de reconocimiento positivos que les generan sentimientos de orgullo, alegría, optimismo, confianza en sí mismos y en el futuro y felicidad. Los resultados también nos indican que las experiencias reconstruidas son usadas para conectar significados sobre sí mismos en distintos ámbitos de la actividad. Dicho de otro modo, los significados no sólo se conectan con sus características como aprendices, sino que también se utilizan para reconocerse como madres, o como hijo, o como electricista, etc. Esto se explica porque como se comentó previamente, la identidad es funcional a los contextos de actividad en los que el sujeto participa. Black et al. (2010) han propuesto el concepto de "leading identity" siguiendo la idea propuesta por Leontiev de "leading activity" para explorar la relación entre las actividades significativas implicadas con el modo como los estudiantes reflexionan sobre su proceso de aprendizaje. Una "leading activity” es, según Leontiev (1981 en Black et al., 2010) una actividad significativa para el sujeto porque en ella emergen nuevos motivos para participar. Para Black et al. (2010), la "leading identity" surge en estas actividades, en estos momentos de emergencia de motivos, permitiendo al sujeto tomar conciencia de sus motivos y organizarlos jerárquicamente.

Junto con el movimiento en los motivos que puede derivar de nuevas "leading activities", se produce en el sujeto un cambio en el modo cómo interpreta y valora las actividades de aprendizaje en las que está involucrado. Por ejemplo, volviendo al caso de Ana, de nuestra primera viñeta, vemos cómo el hecho de sentirse competente en un contexto desafiante (taller mecánico) la lleva a reorganizar sus motivos y decidir retomar su educación formal. En la misma línea es que logra establecer conexiones entre esa experiencia emocionalmente positiva y la disposición optimista con la que enfrentará el nuevo contexto. Para que ocurra este movimiento y el sujeto logre utilizar significados positivos de sí mismo como aprendiz en nuevos contextos, se necesita de experiencias de reconocimiento positivas (reconocimiento que ocurren en la interacción con los demás, y también como alguien que posee ciertas características específicas).

\section{DISCUSIÓN}

A la luz de los resultados obtenidos surge la necesidad de reflexionar sobre el tema de los motivos y el rol de los otros en los procesos de reconocimiento. A su vez, surge la pregunta por las implicancias éticas y pedagógicas que se desprenden de la conceptualización de reconocimiento y alteridad propuestas en los procesos de re-construcción de la identidad de aprendiz. Estas reflexiones permiten profundizar la comprensión sobre el modo cómo los aprendices atribuyen sentido a sus aprendizajes.

En primer lugar, interesa reflexionar sobre el tema de la construcción de motivos de los aprendices adultos. En la introducción se dijo que uno de los grandes temas de esta época 
consiste en fomentar procesos de aprendizaje a lo largo de toda la vida. Esto tiene como consecuencia, que aquellos sujetos que por diferentes razones han quedado marginados de procesos educativos formales, encuentran en la vida nuevos espacios donde aprender y crecer.

Las instancias formativas que buscan re-enganchar a quienes han dejado la escuela o quienes llevan años fuera de sistemas formales, necesitan conectar con las necesidades y motivos de esas personas. Cuestión que remite directamente al tema de la Identidad de Aprendiz. En la medida en que ampliamos la pregunta por qué tipo de personas queremos formar, emerge inmediatamente la contra-pregunta: ¿qué tipo de formación y experiencias previas traen estos aprendices que buscamos reinsertar? Es decir, se visibiliza que para que el aprendizaje sea significativo y transformador, se requiere acoger la historia de ese sujeto y conectar con sus motivos y aprendizajes previos. Ya lo comentaba Freire:

La educación auténtica, repetimos, no se hace de A para B o de A sobre B, sino A con $\mathrm{B}$, con la mediación del mundo. Mundo que impresiona y desafía a unos y a otros originando visiones y puntos de vista en torno de él. Visiones impregnadas de anhelos, de dudas, de esperanzas o desesperanzas que implican temas significativos, en base a los cuales se constituirá el contenido programático de la educación (2005: 68).

A la luz de los resultados, se ve que en la reconstrucción de motivos que los sujetos narran, el aprendizaje va adquiriendo cada vez más sentido cuando se encuentra alineado a proyectos vitales que los entusiasman o ilusionan. Un futuro mejor, un modo de salir de la pobreza, una esperanza de transformar su situación familiar. Ahora bien, ¿este lugar depende solamente de ellos como sujetos aprendices? Vale la pena decir que en este proceso de recoger la subjetividad de los adultos que nuevamente se posicionan como aprendices, emerge el tema del reconocimiento y de cómo el otro tiene el potencial de entregar actos de reconocimiento positivos y negativos que influyen profundamente en ellos. En este punto, y en segundo lugar, vale la pena abordar la importancia del reconocimiento y vincularlo con un "argumento de alteridad" (Peñalver, 2000) que explore el proceso de construcción de identidad de aprendiz. La identidad, desde la actividad, el discurso y situada en una práctica, ofrece un marco de discusión que se ensancha conceptualmente cuando se piensa con pensadores de la ética de la alteridad, como Emmanuel Lévinas.

En particular cuando se trabaja con temas que indagan el modo en que las personas se reconocen y sitúan permite mostrar la relevancia de la intersubjetividad. Se visualiza la relación entre el sujeto y el otro sin perder de vista el lugar que ocupa este último. Desde una perspectiva contemporánea y levinasiana, aquí se disponen alcances éticos fuera de la cuestión imperativa. Se armoniza con un concepto de lo ético que no es definido a partir de una Ley, sino que de un sentido que orienta hacia lo Otro, hacia el lugar que el otro ocupa. Nos referimos al Otro para dar cuenta de ese fenómeno de alteridad que no puede ser subsumido por un Sujeto cerrado; el Otro implica un lugar único y propio. De esta manera queda explicitado que todo otro en su concretitud puede revestir ese lugar único y diferente el cual no está determinado ni definido por una Sujeto universal clausurado (Lévinas, 2006). Lévinas no pretende definir la cuestión ética, sino que dar cuenta de su sentido (Lévinas, 2000). Este sentido radica en el darle un lugar al otro, reconocer su aparición en tanto acontecimiento que irrumpe. La apuesta levinasiana está en que este otro que está 
frente a nosotros empíricamente, articula un sentido ético más allá de su rostro concreto pues impone sobre mí una exigencia infinita de tener que responderle (Orange, 2012). Este andamiaje teórico filosófico nos da elementos para repensar la subjetividad; ahora el otro abre el sentido hacia la subjetividad y re articula las disposiciones intersubjetivas (Lévinas, 2000; Castro-Serrano, 2009). La potencia de la subjetividad no puede pensarse, por lo tanto, sin la idea de alteridad.

Creemos que todo lo anterior es relevante cuando volvemos al contexto que nos convoca: a una investigación educativa con personas en contextos de exclusión. Es relevante pues lo anterior nos invita a no pensar en "La Ética", en esa de lo imperativo como se dijo, sino en una considerada como posición frente a la investigación que permite una ética múltiple (Karsz, 2004; 2007), de la construcción con otros en su aventura y riesgo. "(La ética) Ha de ser inventada con motivo de situaciones por definición singulares y de intervenciones por definición únicas" (Karsz, 2007, p. 203). Es decir, no se comienza desde concepciones neutrales sobre la subjetivación de los participantes de nuestro estudio. Se concuerda con Cabrolié cuando plantea que:

La modernidad en América Latina comienza así, con la negación de la alteridad, con la invisibilización del Otro, de su rostro, de su voz, de su subjetividad. La postmodernidad en cambio, está marcada por la emergencia de la diversidad de múltiples sujetos, abriendo la posibilidad de la interpelación del Otro negado, que para el caso de América Latina tiene múltiples rostros, acaso los más evidentes sean el indígena, la mujer, los pobres, sin desconocer que existen muchos más (2010: 318).

A partir de este trazado, de esta ética que hace irrumpir al otro, volvemos a mirar nuestros resultados. Una ética que reconoce, una ética que refiere a su sentido desplegado a partir del otro, nos saca de una totalidad puramente axiológica en búsqueda del fundamento, cuestión fácilmente confundible con el bien y el mal, con una moralidad estática. Una ética de lo divergente, como la aquí propuesta, permite reflexionar sobre la importancia de darle un lugar al aprendiz (un espacio de subjetivación propia donde el sentido comienza desde el otro significativo) según cada situación que demanda la intervención educativa en contextos de exclusión y pobreza.

En otras palabras, una parte importante de preguntarse por el modo cómo se reconocen los sujetos en tanto aprendices, tiene relación con las posibilidades contextuales y sociales que tienen disponibles para realizar este proceso. No se trata, pues, ya solo de la micro-génesis de las experiencias de reconocimiento -importantes para todos en tanto seres humanos y sociales-, sino también del entramado de sistemas de distinto orden (Brofrenbrenner, 1987) que posibilitan o entorpecen, que en definitiva mediatizan el proceso de construir significados en cuanto aprendiz. Lo que está en juego no es, por tanto, si se cuenta con oportunidades de aprendizaje, sino más radicalmente, si se disponen de experiencias de reconocimiento para poder construir sentido en el aprendizaje desde esa nueva subjetividad reconocida. Esto, además, pone el acento en las posibilidades que se encuentran para ir reconstruyendo las experiencias en el marco de las trayectorias de aprendizaje.

Lo revisado en este estudio muestra la importancia de la identidad del aprendiz para significar el aprendizaje, una herramienta conceptual que permite situarse y pensarse. Pero, como ya se dijo, lo que posibilita este proceso es el hecho de ser reconocidos por otro a través 
de la participación en actividades concretas. En este proceso, las relaciones emocionales, los vínculos significativos impulsan y configuran posibilidades de reconocimiento. Los otros significativos, principalmente familiares y, como vimos en este estudio, también maestros y compañeros, abren nuevos modos de disponerse y de encontrarse frente al aprendizaje. Permiten al sujeto construir significados y atribuir sentido a su participación, a través de la reorganización de los motivos de aprendizaje.

Además de la participación en situaciones cotidianas, se reconstruye la identidad de aprendiz cuando se cuenta con un dispositivo discursivo cuya meta es la reflexión sobre nosotros como aprendices y sobre el modo como enfrentamos las actividades de aprendizaje (Falsafi, 2010). Estas actividades discursivas guiadas no son frecuentes en nuestros contextos educativos actuales. Pero de vez en cuando, ocurren, permitiendo al sujeto utilizar nuevas herramientas conceptuales en el sentido vigotskiano del término. De este modo, en las situaciones en que relatamos a otros nuestras trayectorias como aprendices, se generan estos dispositivos que permiten la co-construcción de significados sobre la manera en que nos reconocemos. Como Ramírez (2014) señala, "Los relatos de vida son una reflexión sobre la propia experiencia, es la mirada sobre sí para entender las decisiones tomadas, para dar sentido a las situaciones (...)", sin embargo, "Esta reflexión no pone el foco solo en la propia acción, sino también en la acción de los demás respecto a la propia persona o respecto a otros" (2014: 125-126).

Volviendo a la lectura ética, se aprecia un tránsito en la subjetivación desde la experiencia de exclusión hacia un espacio validado de aprendiz el cual es reconocido mediante otros; ahí sobresale un espacio de unicidad en ese marco de aprendizaje. Le permite situarse en el mundo desde su lugar de único (Ramírez, 2014; Lévinas, 2001). Aquí hace eco el caso de Ana, el primer ejemplo, que refiere que ahora con su título de enseñanza secundaria completa: "como que hasta el trato cambió". Hay un movimiento recursivo y dinámico entre un posicionamiento diferente y una experiencia de que te traten (y reconozcan) de otra manera (como única). En este proceso cabría entonces la posibilidad de reconocerse como alguien capaz de aprender, capaz de generar conocimiento y subjetivarse gracias al lugar que se co-construye junto a otros (Rubilar, 2013).

Se visualiza, de este modo, la importancia de una ética del reconocimiento que comprende la relación individual-colectiva y el lugar que ocupa el reconocimiento al interior de una sociedad justa (Castro-Serrano et al., 2014). En esta dirección se camina cuando podemos pesquisar las funciones de "los otros" en la reconstrucción de la identidad de aprendiz. En este punto, los alcances del otro nos ayudan a entender la significatividad de las experiencias clave de aprendizaje. Como veíamos en los resultados, la mayoría de las experiencias clave se asocian a emociones positivas en las que los otros juegan un rol potente entregando actos de reconocimiento generalmente positivos. Esta significación emocional cruza estas experiencias y permite a los sujetos pensarse, reconocerse y situarse hacia el pasado y hacia el futuro.

El modelo de Identidad de Aprendiz entiende que la identidad es permanentemente co-construida con otros. Las ideas de Lévinas van en la misma dirección al proponer una noción de alteridad que fisura y rompe la Identidad como consciencia de todo (Lévinas, 2006; 2000). Esto nos invita a repensar la importancia del lugar de la intersubjetividad en los contextos educativos. La filosofía de Lévinas invita a crear un modo de hacer y convivir en el contexto educativo repensando los modos de subjetivación para el aprendizaje y el rol significativo que juega el docente para otorgar reconocimiento (Ramírez, 2014). El 
concepto de experiencia clave de aprendizaje, permite a su vez, poner el acento en el tema de las emociones, los motivos y el reconocimiento, posibilitando el análisis del rol de los otros en los procesos previos de significación del aprendizaje.

Dicho todo lo anterior, se hace necesario volver a la pregunta por la atribución de sentido del aprendizaje. Como ya ha sido expresado claramente por Coll (1988; 2003; 2014): "La atribución de sentido a los contenidos y las actividades de aprendizaje es un proceso complejo en el que intervienen múltiples factores, tanto de tipo cognitivo como, muy especialmente, de tipo emocional y relacional" (2003: 5). En este complejo proceso, vale la pena recordar que las personas son capaces de atribuir sentido al esfuerzo por aprender no sólo en la medida en que quieren, sino en la medida en que pueden. Por lo tanto, identificar las experiencias clave de aprendizaje de los sujetos puede ayudar a hacer visibles para ellos aquellas experiencias a las que han otorgado más significancia y conectarlas con los motivos de sus contextos de aprendizajes actuales. Estas instancias de encuentro permiten, por lo tanto, la reconstrucción de las experiencias previas posibilitando nuevos procesos de atribución de sentido. Lo anterior instala una trama ética, y necesaria si aspiramos a que los adultos con trayectorias de abandono escolar y de exclusión social logren encontrar nuevos espacios de resignificación y se fortalezcan como aprendices, y en definitiva, como sujetos.

\section{REFERENCIAS BIBLIOGRÁFICAS}

Barnett, R. (2011). Lifewide Education: A Transformative Concept for Higher Education. In N. Jackson (Ed.), Learning for a Complex World (pp. 22-38). USA: Authorhouse.

Biesta, G.J.J., Field, J., Hodkinson, P., Macleod, F.J. \& Goodson, I.F. (2011). Improving learning through the lifecourse: Learning lives. London/New York: Routledge.

Black, L., Williams, J., Hernandez-Martinez, P., Davis, P. \& Wake, G. (2010). Developing a 'Leading Identity': The relationship between students' mathematical identities and their career and higher education aspirations. Educational Studies in Mathematics, vol.73 (1), 55-72. DOI 10.1007/ s10649-009-9217-x

Bronfenbrenner, U. (1987). La ecología del desarrollo humano. Barcelona: Paidós Transiciones.

Cabrolié, M. (2010). La intersubjetividad como sintonía en las relaciones sociales. Redescubriendo a Alfred Schütz. Polis. Revista de la Universidad Bolivariana, vol.9 (27), 317-327.

Castro-Serrano, B., Flotts de los Hoyos, M., Valenzuela Parada, C., y Vidal Molina, P. (2014). Bienestar subjetivo y pobreza: Estudio de caso con mujeres en Santiago de Chile. Ánfora, vol.21 (37), 129-150.

Castro-Serrano, B. (2009). Las posibilidades del sentido y la alteridad radical: un recorrido arqueológico por el pensamiento de Lévinas, Daimon, 48, 81-96.

Coll, C. (1988). Significado y sentido en el aprendizaje escolar. Reflexiones en torno al concepto de aprendizaje significativo. Infancia y Aprendizaje, 41, 131-142.

Coll, C. (2003). Esfuerzo, ayuda y sentido en el aprendizaje escolar. Aula de Innovación Educativa, $120,37-43$.

Coll, C. (2014). El sentido del aprendizaje hoy: un reto para la innovación educativa. Aula de Innovación Educativa, 232, 12-17.

Coll, C. \& Falsafi, L. (2010). Learner Identity - an educational and analytical tool. Revista de Educación, 353, 211-233.

Delors, J. (Ed.) (1996). La educación encierra un tesoro. Madrid: Santillana - Ediciones UNESCO.

Denzin, N. (1989). Interpretive Biography. Newbury Park: Sage Publications. 
Falsafi, L. (2010). Learner Identity a sociocultural approach to how people recognize and construct themselves as learners. (Tesis inédita de doctorado). Universidad de Barcelona, Barcelona, ES.

Freire, P. (2005). Pedagogía del oprimido. México: Siglo XXI.

Gee, J.P. (2000). Identity as an analytic lens for research in education. Review of Research in Education, 25, 99125.

González-Rey, F. (2010). Las categorías de sentido, sentido personal y sentido subjetivo en una perspectiva histórico-cultural: un camino hacia una nueva definición de subjetividad. Universitas Psychologica, vol.9 (1), 241-253.

Honneth, A. (1997). La lucha por el reconocimiento. Por una gramática moral de los conflictos sociales. Barcelona: Editorial Crítica.

Karsz, S. (2004). La exclusión bordeando sus fronteras. Definiciones y matices. Barcelona: Gedisa.

Karz, S. (2007). Problematizar el Trabajo Social: definiciones, figuras, clínica. Barcelona: Gedisa.

Lave, J., \& Wenger, E. (1991). Situated learning: Legitimate peripheral participation. New York: Cambridge University Press.

Lévinas, E. (2000). Ética e Infinito. Madrid: La balsa de la Medusa.

Lévinas, E. (2001). Entre nosotros, Ensayos para pensar en otro. Valencia: Pre-textos.

Lévinas, E. (2003). Humanismo del otro hombre. México: Siglo XXI.

Lévinas, E. (2006). Totalidad e infinito. Ensayo sobre la exterioridad. Salamanca: Sígueme.

Leontiev, A. (1978). Activity, consciousness, and personality. Englewood Cliffs, NJ: Prentice Hall.

Leontiev, A. (2005). Lecture 13. The structure of consciousness. Journal of Russian and East European Psychology, vol.43 (5), 14 - 24.

McAdams, D.P., Josselson, R. \& Lieblich, A. (Eds.). (2006). Identity and story: Creating self in narrative. Washington, DC: APA.

Orange, D. (2012). Pensar la práctica clínica. Recursos filosóficos para el Psicoanálisis Contemporáneo y las Psicoterapias Humanistas. Santiago de Chile: Cuatro Vientos.

Peñalver, P. (2000). Argumento de Alteridad, Madrid: Caparrós.

Ramírez, J. (2014). Personas extraordinarias en la trayectoria de vida de dos docentes: mirada del otro y sensibilidad moral en el vínculo. Perspectiva Educacional. Formación de Profesores, vol.53 (2), 112-128. DOI 10.4151/07189729-Vol.53-Iss.2-Art.279

Rubilar, G. (2013). Imágenes de Alteridad. Reflexiones y aportes para el trabajo social en contextos de pobreza y exclusión. Santiago de Chile: Editorial Universidad Católica.

Sfard, A. \& Prusak, A. (2005). Telling identities: In search of an analytic tool for investigating learning as a culturally shaped activity. Educational Researcher, vol.34 (4), 14-22. DOI https:// doi.org/10.3102/0013189X034004014

Willig, C. (2013). Introducing Qualitative Research in Psychology. Berkshire: Open University Press.

Wortham, S. (2004). From good student to outcast: The emergence of a classroom identity. Ethos, vol.32 (2), 164-187.

Woods, P. (1997). Experiencias críticas en la enseñanza y el aprendizaje. Barcelona: Paidós.

Yair, G. (2008). Key Educational Experiences and Self-Discovery in Higher Education. Teaching and Teacher Education: An International Journal of Research and Studies, vol.24 (1), 92-103. DOI https://doi.org/10.1016/j.tate.2007.04.002 
